\title{
Networking Particles Over Distance Using Modular Oligonucleotide-based Devices
}

\author{
Ruslan Yashin ${ }^{\ddagger}$, Sergei Rudchenko***, and Milan N. Stojanovic*t
}

\section{SUPPORTING INFORMATION}

\section{General:}

Experimental procedures and sequences for experiments described in the article:

DNA Sequences. All DNA strands were purchased from Integrated DNA Technologies, Inc. and TriLink BioTechnologies and purified by PAGE \& HPLC. DNA stock solutions ( $1 \mu \mathrm{M}$ and $100 \mu \mathrm{M})$ were prepared in DEPC-Treated Water (Ambion) and stored at $-20{ }^{0} \mathrm{C}$ before use. The sequences used in the manuscript are as follows.

Strand: Enzyme $\mathbf{E}_{1}$

Sequence:

5' (Biotin-TEG) TTT TTT TTT TCG GAG AAG AGG TGT TCG GCT TGA GAC TCT TCT CCG AGC CGG TCG AAA TAG TGA -3'

Strand: Enzyme $\mathbf{E}_{\mathbf{2}}$

Sequence:

5’ -/5BioTEG/TTT TTT TTT TTT TTT TTT TTT CTC TTC TCC GAG CCG GTC GAA ATA GTG A -3’

Strand: Enzyme $\mathbf{E}_{\mathbf{3}}$

Sequence:

5' -/5BioTEG/TTT TTT TTT TTT TTT TTT TTT CTC TTC TCC GAG CCG GTC GAA ATA GTG A -3'

Strand: Input 1 (I1)

Sequence:

5' -/5BioTEG/ TCT CAA GCC GAA CAC CT -3'

Strand: Substrate $\mathbf{S}_{\mathbf{1}}$

Sequence:

5'- /6-FAM/TTG GCG TTA ACT CCA TCA CTA TrAG GAA GAG/3BioTEG/ -3'

Strand: Substrate $\mathbf{S}_{\mathbf{2}}$

Sequence:

5'-15Cy5/GTC TCA AGC CGA ACA CCT TCA CTA TrAG GAA GAG TTT TTT TTT TTT /3BioTEG/ -3'

Strand: oligonucleotide $\mathbf{C}_{\mathbf{O}}$

Sequence:

5'- /5Bio/ TGG AGT TAA CGC CAA TGC GAC -3'

Strand: oligonucleotide $\mathbf{C}_{\mathbf{I 2}}$

Sequence:

5' - AAA ACT CTT CCT ATA GTG AAG GTG CCG TAC GGA-3'

Strand: Input 2 (I2)

Sequence:

5' - TCC GTA CGG CAC CTT CAC TAT AGG AAG AGT TTT-3'

Strand: oligonucleotide $\mathbf{C}_{03}$

Sequence:

5'- CTC TTC CTA TAG TGA TGG AGT ACC TTG ACG T -3'

Strand: oligonucleotide $\mathbf{C}_{\mathbf{1 3}}$

Sequence:

5'-TAT TTC GAC CGG CTC GGA CCG TCC -3'

Strand: Input 3 (I3)

Sequence:

5'- GGA CGG TCC GAG CCG GTC GAA ATA -3'

Strand: Substrate $\mathbf{S}_{\mathbf{3}}$

Sequence:

5'-15Cy5/ (C6-NH)ACG TCA AGG TAC TCC ATC ACT ATA GGA AGA GTC AC TA TrAG GAA GAG TTT /3BioTEG/ -3' 
Microspheres (beads) used in the manuscript are as follows:

$\mathbf{D}_{2}$.

\begin{tabular}{|c|c|c|}
\hline Beads: Streptavidin C & bated C & ELTM Magnetic (Bangs Laborator \\
\hline Biotin binding capacity & -0.23 & mg biotin-FITC/mg microspheres \\
\hline Mean Diameter: & -2.8 & $\mu \mathrm{m}$ \\
\hline
\end{tabular}

$\mathbf{D}_{1}$ or $\mathbf{A N D}$ or $\mathbf{A}_{2}$.

\begin{tabular}{|lll|}
\hline \multicolumn{2}{|c|}{ Beads: Streptavidin Coated COMPELTM Magnetic (Bangs Laboratories, Inc.) } \\
Biotin binding capacity: & -0.11 & $\mathrm{mg}$ biotin-FITC/mg microspheres \\
Mean Diameter: & -5.8 & $\mu \mathrm{m}$ \\
\hline
\end{tabular}

$\mathbf{A}_{1}$

\begin{tabular}{|lll|}
\hline Beads: Streptavidin Coated COMPELTM Magnetic (Bangs Laboratories, Inc.) \\
Biotin binding capacity: & -0.038 & $\mathrm{mg}$ biotin-FITC/mg microspheres \\
Mean Diameter: & -8.27 & $\mu \mathrm{m}$ \\
\hline
\end{tabular}

$\mathbf{D}_{3}$.

Beads: Streptavidin Coated COMPELTM Latex (Bangs Laboratories, Inc.)

Biotin binding capacity: $\quad-0.068 \quad \mathrm{mg}$ biotin-FITC/mg microspheres

Mean Diameter: $\quad-5.60 \quad \mu \mathrm{m}$

Preparation of microspheres. Necessary volume of beads from stock solution was washed 3 times by 0.7 $\mathrm{ml}$ of washing buffer (WB) [0.1 M NaCl, 20mM Tris (pH 7.5), $5 \mathrm{mM} \mathrm{KCl,} 0.1 \mathrm{mg} / \mathrm{ml} \mathrm{BSA}]$

(centrifugation : $5 \mathrm{~min}$ at 14 000). Washed beads were then conjugated with DNA at room temperature in incubating buffer (IB) $[1 \mathrm{M} \mathrm{NaCl}, 20 \mathrm{mM}$ Tris , $0.1 \mathrm{mg} / \mathrm{ml} \mathrm{BSA}]$ according to the plan below.

For the system with AND hub.

First incubation.

$25000000-2.8 \mu \mathrm{m}$ magnetic beads were 1.5 hours incubated with $45 \mathrm{nM} \mathrm{\mathbf {S } _ { 2 }}(\mathrm{V}=400 \mu \mathrm{l})$.

$1500000-5.8 \mu \mathrm{m}$ magnetic beads were 1.5 hours incubated with $21 \mathrm{nM} \mathrm{S} \mathbf{S}_{\mathbf{1}}(\mathrm{V}=200 \mu \mathrm{l})$.

200000 - $8.2 \mu \mathrm{m}$ magnetic beads were overnight incubated with $50 \mathrm{nM} \mathbf{C}_{\mathbf{0 1}}(\mathrm{V}=100)$.

15000000 - $5.6 \mu \mathrm{m}$ latex beads were 1.5 hours incubated with $37 \mathrm{nM} \mathrm{S}_{\mathbf{3}}(\mathrm{V}=400 \mu \mathrm{l})$.

Beads were washed 3 times with WB buffer

Second incubation

25000000 - $2.8 \mu \mathrm{m}$ magnetic beads were 2 hours incubated with $2.5 \mu \mathrm{M} \mathbf{C}_{\mathbf{I 2}}$.

1500000 - $5.8 \mu \mathrm{m}$ magnetic beads were 2 hours incubated with $2.5 \mu \mathrm{M} \mathbf{C}_{\mathbf{0 3}}$.

15000000 - $5.6 \mu \mathrm{m}$ latex beads were overnight incubated with $1.2 \mu \mathrm{M} \mathbf{E}_{\mathbf{3}}$ and $2.5 \mu \mathrm{M} \mathbf{C}_{\mathbf{I 3}}$.

Beads were washed 3 times with WB buffer

Third incubation

25000000 - $2.8 \mu \mathrm{m}$ magnetic beads were overnight incubated with $1.2 \mu \mathrm{M} \mathbf{E}_{\mathbf{2}}$ and $0.5 \mu \mathrm{M} \mathbf{C}_{\mathbf{I 2}}$.

1500000 - $5.8 \mu \mathrm{m}$ magnetic beads were overnight incubated with $1.2 \mu \mathrm{M} \mathbf{E}_{\mathbf{1}}$ an $0.5 \mu \mathrm{M} \mathbf{C}_{\mathbf{0 3}}$.

15000000 - $5.6 \mu \mathrm{m}$ latex beads were overnight incubated with $1.2 \mu \mathrm{M} \mathbf{C}_{\mathbf{I 3}}$.

Beads were washed 3 times with WB buffer

Latex beads were washed at $4{ }^{0} \mathrm{C}$.

Fluorescence Data. Fluorescence data was acquired on a FACSCalibur flow cell-based benchtop

cytometer (BD Biosciences). Beads from lower part of transwell were diluted to a final volume of $500 \mu \mathrm{l}$ and beads from upper part were diluted to a final volume of $400 \mu \mathrm{l}$ at room temperature and were excited at 488 Blue Laser and 635 Red Diode Laser. 


\section{Quantification of oligonucleotide release and capture on each level of a chain.}

\section{First layer:}

$2.8 \mu \mathrm{m}$ polystyrene magnetic beads

We take 6250000 beads, and this amount binds approximately 38 pmol of biotin based on information provided by manufacturer. After the incubation beads bind 4 pmol of substrate $\mathbf{S}_{\mathbf{2}}$ (based on the removal of fluorescence from solution). Then, the beads are saturated at remaining biotin-binding sites by enzyme $\mathbf{E}_{2}$, estimated at 34 pmol. Based on calibrated change in fluorescence upon release, we estimate that $70 \%$ or 2.8 pmol of $\mathbf{O}_{2}$ leaves the surface of these beads.

\section{$5.6 \mu$ latex beads}

We take 3750000 beads, and this amount binds approximately $31 \mathrm{pmol}$ of biotin based on information provided by manufacturer. After the incubation beads bind 3.6 pmol of substrate $\mathrm{S}_{3}$ (based on removal of fluorescence from solution). Then, the beads are saturated by enzyme $\mathrm{E}_{3}$, estimated at 27 pmol. Based on calibrated change in fluorescence upon release, we estimate that $70 \%$ or 2.5 pmol of $\mathrm{O}_{3}$ leaves the surface of these beads.

\section{Second layer:}

We know from experimental data (calibrated fluorescence) that approximately $50 \%$ of leaving oligonucleotides reach the target on the second layer. Thus, the second layer captures about 1.3 pmoles of oligonucleotides released from the first layer.

$5.8 \mu \mathrm{m}$ polystyrene magnetic beads

We take 500000 beads, and this amount binds approximately 7.4 pmol of biotin based on information provided by manufacturer. After the incubation beads bind 1 pmol of substrate $S_{1}$ (based on removal of fluorescence from solution). Then, the beads are saturated by enzyme $\mathrm{E}_{1}$, estimated at 6.4 pmol. Based on calibrated change in fluorescence upon release, we estimate that $50 \%$ or 0.5 pmol of $\mathrm{O}_{1}$ leaves the surface of these beads.

\section{Third layer.}

We know from experimental data (calibrated fluorescence) that approximately $50 \%$ of leaving oligonucleotides reach the target on third layer. So the third layer captures about 0.3 pmoles of oligonucleotides released from the second layer.

\section{$8.2 \mu \mathrm{m}$ polystyrene magnetic beads}

We take 50000 beads, and this amount binds approximately 7.4 pmol of biotin based on information provided by manufacturer. After the incubation beads bind 7.4 pmol of oligonucleotide $\mathrm{C}_{\mathrm{O} 1}$. 
Results for communication between two microspheres separated by a membrane:

Sequences are the same as in the text.
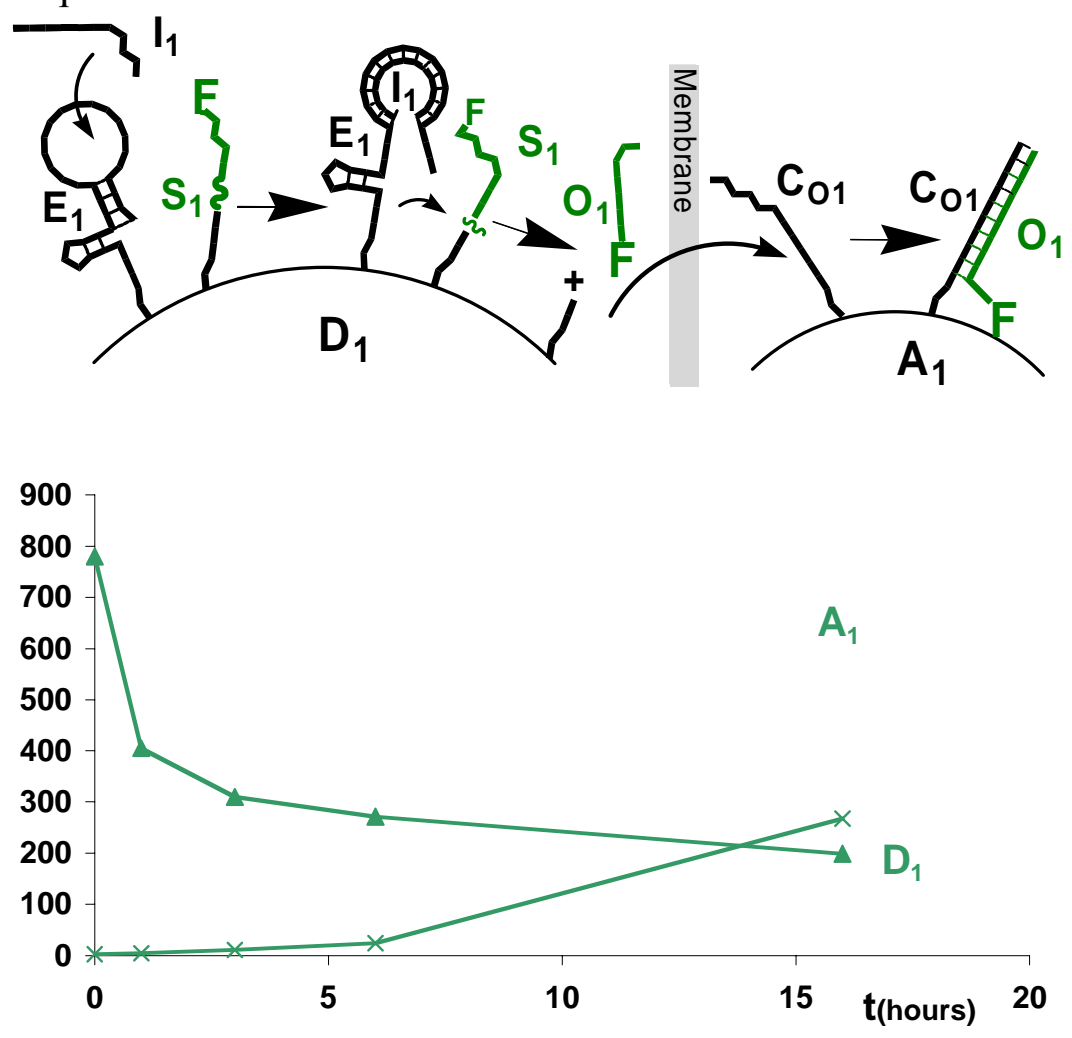

Figure S1. a. Communication between two particles over distance separated by a membrane (Donor-acceptor cascade): Donor bead $\left(\mathbf{D}_{1}\right)$ sense stimuli $\left(\mathbf{I}_{1}\right)$, releasing oligonucleotide signals $\mathbf{O}_{1}$ This is accomplished by coating the bead with catalytic nucleic acid enzyme $\mathbf{E}_{1}$ sensitive to $I_{1}$ and substrate $\mathbf{S}_{1}$. $\mathbf{O}_{1}$ is captured by $\mathbf{A}_{1}$ bead covered with its complement $\mathbf{C}_{\mathrm{O} 1}$. b. Time course (relative fluorescence vs. time) of communication between two particles over distance separated by a membrane.

The $\mathbf{D}_{\mathbf{1}}$ bead performs a YES logic operation with $\mathbf{I}_{\mathbf{1}}$ as inputs, and oligonucleotide $\mathbf{O}_{\mathbf{1}}$ as an output $\left(\mathbf{D}_{1}, 5.8 \mu \mathrm{m}\right)$ through the following chemistry: $\mathbf{I}_{\mathbf{1}}$ activates catalytic molecular beacon $\mathbf{E}_{\mathbf{1}}$ (Figure 1a). In the next step, oligonucleotide $\mathbf{O}_{1}$ is captured by $\mathrm{A}_{1}$ bead $(8.2 \mu \mathrm{m})$. 
Results for three layer cascade with microspheres separated by a membreane

Sequences are the same as in the text.

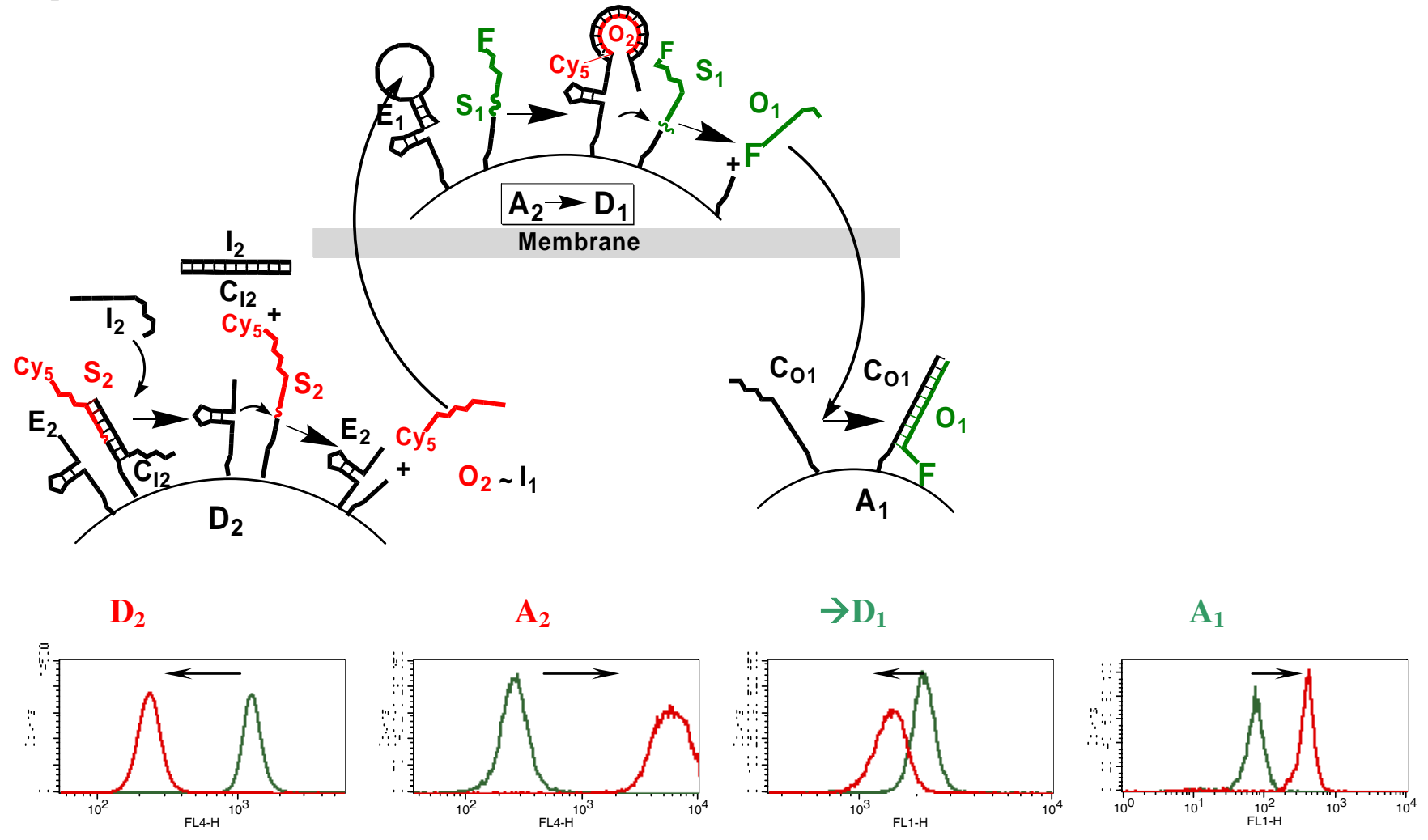

Figure S2. The schematic representation of three-microparticle network with three beads, with a central bead acting as YES gate: $\mathbf{a}$. Bead $\mathbf{D}_{\mathbf{2}}$ report the presence of oligonucleotides $\mathbf{I}_{\mathbf{1}}$ to the hub bead $\mathbf{A}_{\mathbf{2}} \rightarrow \mathbf{D}_{\mathbf{1}}$ by releasing Cy5labeled oligonucleotide $\mathbf{O}_{2}$. The YES gate releases fluorescein labeled oligonucleotide $\mathbf{O}_{\mathbf{1}}$ which is then accepted by $A_{1}$. b. Flow cytometry results, color coding: red $-\mathbf{1}$ added, green - none added. $\mathbf{D}_{2}$ panel show decrease of fluorescence due to release of Cy5 labeled $\mathbf{O}_{2} ; \mathbf{A}_{2} \rightarrow \mathbf{D}_{1}$ panel show increase of fluorescence due to transfer of oligonucleotide $\mathbf{O}_{2} ; \mathbf{A}_{\mathbf{2}} \rightarrow \mathbf{D}_{1}$ panel shows decrease in fluorescence due to release of fluorescein labeled oligonucleotide $\mathbf{O}_{\mathbf{1}} ; \mathbf{A}_{\mathbf{1}}$ panel shows an increase in fluorescence upon binding of oligonucleotide $\mathbf{O}_{\mathbf{1}}$.

The first layer of bead $\left(\mathbf{D}_{2}, 2.8 \mu \mathrm{m}\right)$ senses $\mathbf{I}_{\mathbf{2}}$, and subsequently releases oligonucleotide $\mathbf{O}_{2}$. The bead is covered with enzyme and Cy5-labeled substrate $\left(\mathbf{E}_{\mathbf{2}}\right.$ cleaves $\mathbf{S}_{\mathbf{2}}$ on $\left.\mathbf{D}_{\mathbf{2}}\right)$. On the $\mathbf{D}_{\mathbf{2}}$ bead substrate is blocked with $\mathbf{C}_{\mathbf{I} 2}$ complementary to $\mathbf{I}_{2}$ (Figure 2). The sensor bead is activated through a stranddisplacement-based recognition: $\mathbf{I}_{\mathbf{2}}$ recognizes $\mathbf{C}_{\mathbf{I} 2}$ on $\mathbf{D}_{\mathbf{2}}$. $\mathbf{I}_{\mathbf{2}}$ triggers the catalytic release of oligonucleotides $\mathbf{O}_{2}$ (Cy5-labeled), which then serve as input for $\mathbf{A}_{\mathbf{2}} \rightarrow \mathbf{D}_{\mathbf{1}}$ bead. The $\mathbf{A}_{\mathbf{2}} \rightarrow \mathbf{D}_{\mathbf{1}}$ bead performs an YES logic operation with $\mathbf{O}_{\mathbf{2}}$ as inputs, and oligonucleotide $\mathbf{O}_{\mathbf{1}}$ as an output $\left(\mathbf{A}_{\mathbf{2}} \rightarrow \mathbf{D}_{\mathbf{1}}, 5.8 \mu \mathrm{m}\right)$ through the following chemistry: $\mathbf{O}_{\mathbf{2}}$ activates catalytic molecular beacon $\mathbf{E}_{\mathbf{1}}$ (Figure 5). In the final step of this network, oligonucleotide $\mathbf{O}_{1}$ is captured by $\mathrm{A}_{1}$ bead $(8.2 \mu \mathrm{m})$, which shows approximately fivefold increase in fluorescence, only if the $\mathbf{A}_{\mathbf{2}} \rightarrow \mathbf{D}_{\mathbf{1}}$ bead is active. 


\section{Additional experiments with structure switching sensors:}

\section{Experiment 1: Remote sensing of a small molecule analyte (ATP) based on two beads communication system.}

Structure of oligonucleotides used.

Strand: Oligonucleotide 6

Sequence:

5' - /5BioTEG/ TTT TTT TTT TTT ACG TCC ACC TGG GGG AGT ATT GCG GAG GAA GGT

Strand: Oligonucleotide 7

Sequence:

5' - CCC AGG TGG ACG GTC CGA GCC GGT CGA AAT A/3Cy5Sp/ -3’

Strand: Oligonucleotide 7c

Sequence:

5’- /5Bio/ TTT TAT TTC GAC CGG CTC GGA CCG TCC ACC TGG G -3’

4000000 - $5.8 \mu \mathrm{m}$ magnetic beads were overnight incubated with $1 \mu \mathrm{M} 6$ and $2 \mu \mathrm{M} 7$.

1200000 - $8.2 \mu \mathrm{m}$ magnetic beads were overnight incubated with $1 \mu \mathrm{M}$ 7c

a.

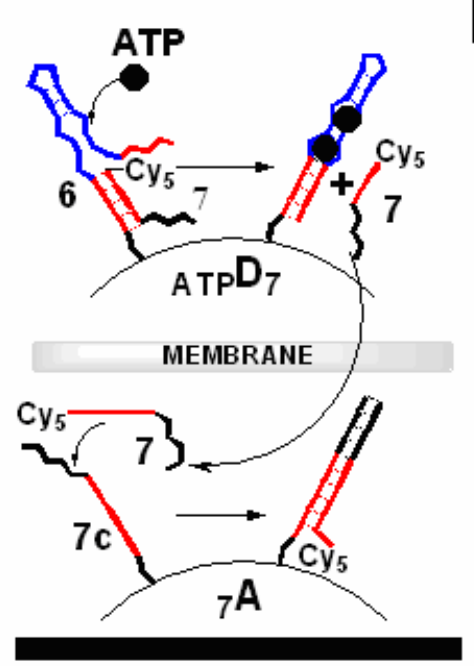

b.

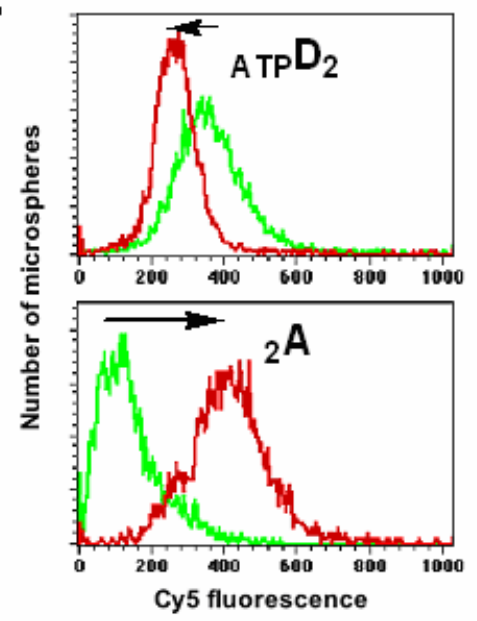

Figure S1. Remote sensing: a. Upon sensing ATP, a donor bead $\left({ }_{\mathbf{A T P}} \mathbf{D}_{7}\right)$ coated with a structure-switching adenosine sensor releases Cy5 labeled, which is captured by the second set of beads ${ }_{7} \mathbf{A}$. b. Upper panel shows the flow cytometry results with donor beads decreasing in Cy5 fluorescence; lower panel shows an increase in fluorescence of acceptor beads. 
Experiment 2: Four-particles network with four beads, and a central bead acting as a hub AND gate and with one bead as sensor for ATP.

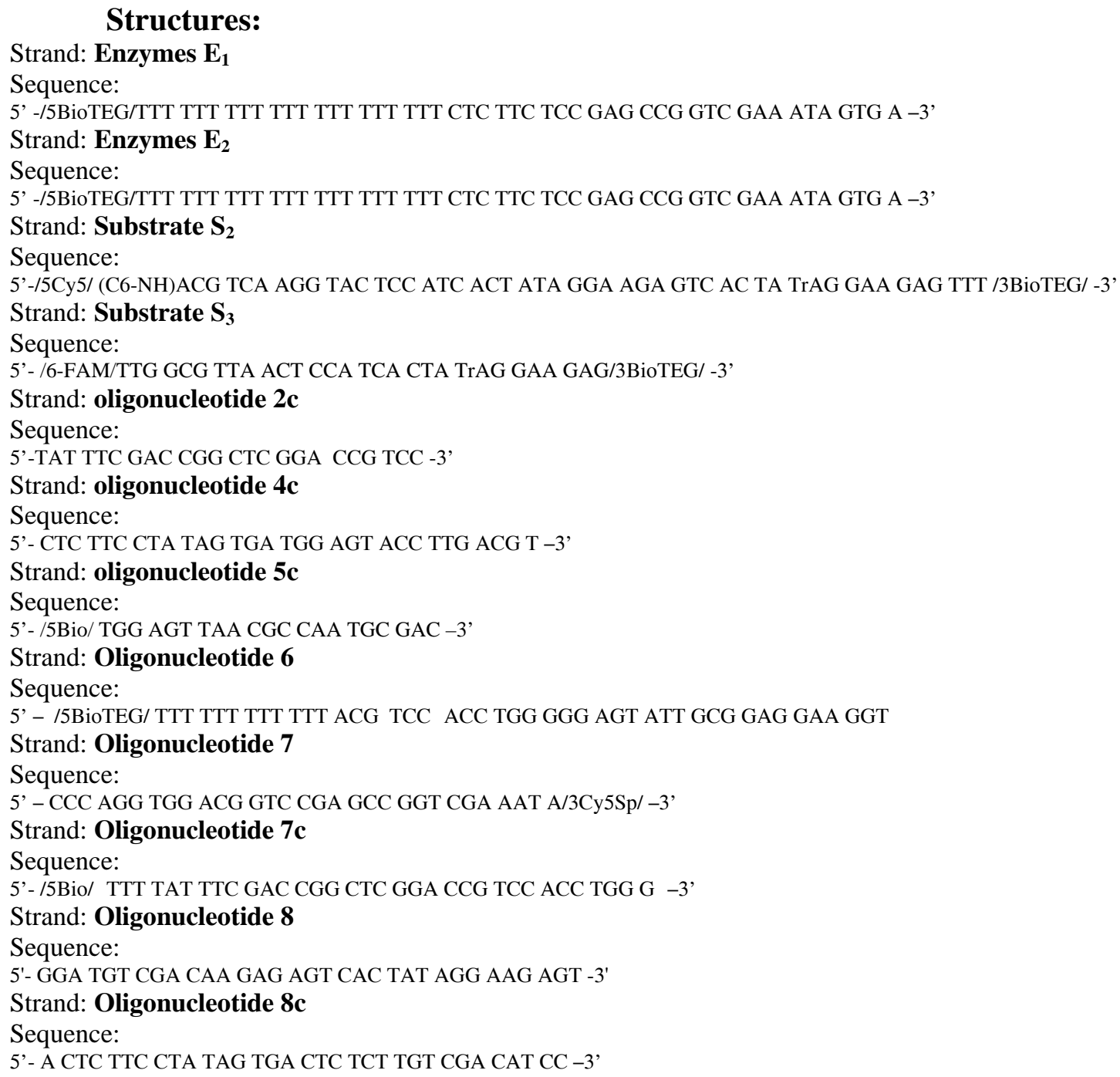

For AND oligonucleotide donor-acceptor system.

First incubation.

25000000 - $2.8 \mu \mathrm{m}$ magnetic beads were 1.5 hours incubated with $45 \mathrm{nM} \mathrm{\mathbf {S } _ { 2 }}(\mathrm{V}=400 \mu \mathrm{l})$.

1500000 - $5.8 \mu \mathrm{m}$ magnetic beads were 1.5 hours incubated with $21 \mathrm{nM} \mathrm{\mathbf {S } _ { 3 }}(\mathrm{V}=200 \mu \mathrm{l})$.

200000 - $8.2 \mu \mathrm{m}$ magnetic beads were overnight incubated with $50 \mathrm{nM} \mathrm{5c}(\mathrm{V}=100)$. 15000000 - $5.6 \mu \mathrm{m}$ latex beads were overnight incubated with $1 \mu \mathrm{M} 6$ and $2 \mu \mathrm{M} \mathrm{7.} \mathrm{(V=200 \mu l).}$ Beads were washed 3 times with WB buffer

Second incubation

25000000 - $2.8 \mu \mathrm{m}$ magnetic beads were 2 hours incubated with $2.5 \mu \mathrm{M} \mathrm{8c}$.

1500000 - $5.8 \mu \mathrm{m}$ magnetic beads were 2 hours incubated with $2.5 \mu \mathrm{M} 4 \mathbf{c}$.

Beads were washed 3 times with WB buffer

Third incubation

25000000 - $2.8 \mu \mathrm{m}$ magnetic beads were overnight incubated with $1.2 \mu \mathrm{M} \mathbf{E}_{\mathbf{1}}$ and $0.5 \mu \mathrm{M} \mathrm{8c}$. 1500000 - $5.8 \mu \mathrm{m}$ magnetic beads were overnight incubated with $1.2 \mu \mathrm{M} \mathrm{E} \mathbf{E}_{2}$ and $0.5 \mu \mathrm{M} \mathrm{4c}$.

Latex beads were washed at $4{ }^{0} \mathrm{C}$ 


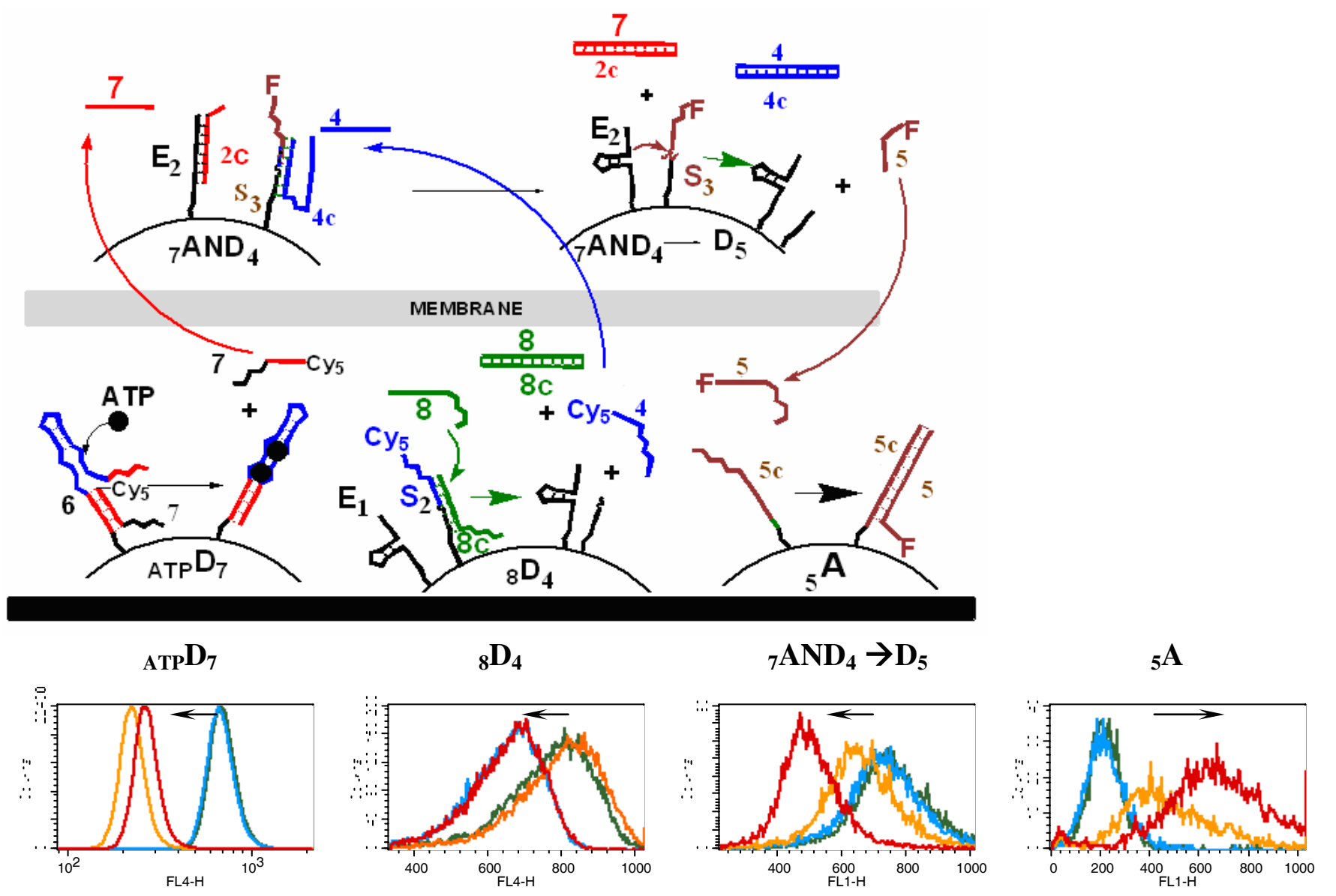

Figure S4. The schematic representation of four-microparticle network with four beads, with a central bead acting as a hub AND gate and with one bead as sensor ATP for: $\mathbf{a}$. Beads ${ }_{\mathbf{A T P}} \mathbf{D}_{\mathbf{7}}$ and ${ }_{\mathbf{8}} \mathbf{D}_{\mathbf{4}}$ report the presence of $\mathbf{A T P}$ and oligonucleotides 8 to the hub bead ${ }_{7} \mathbf{A N D}_{\mathbf{4}} \rightarrow \mathbf{D}_{5}$ by releasing Cy5-labeled oligonucleotides $\mathbf{7}$ and $\mathbf{4}$. The hub releases fluorescein labeled oligonucleotide $\mathbf{5}$ which is then accepted by ${ }_{5} \mathbf{A}$. b. Flow cytometry results, color coding: red - ATP and 8 added, gold $-\mathbf{8}$ added, blue ATP added, green - none added ${ }_{\mathbf{A T P}} \mathbf{D}_{7}$ and ${ }_{8} \mathbf{D}_{\mathbf{4}}$ panels show decrease of fluorescence due to release of Cy5 labeled $\mathbf{7}$ and $\mathbf{4} ;{ }_{7} \mathbf{A N D}_{\mathbf{4}} \rightarrow \mathbf{D}_{\mathbf{5}}$ panel shows decrease in fluorescence due to release of fluorescein labeled $\mathbf{5} ; 5 \mathbf{A}$ panel shows an increase in fluorescence upon binding of $\mathbf{5}$.

In this system first layer of beads $\left({ }_{\mathbf{A T P}} \mathbf{D}_{7}, 5.6 \mu \mathrm{m}\right.$, latex and ${ }_{\mathbf{8}} \mathbf{D}_{\mathbf{4}}, 2.8 \mu \mathrm{m}$, magnetic) senses ATP and oligonucleotides $\mathbf{8}$, and subsequently releases oligonucleotides $\mathbf{7}$ and $\mathbf{4}$. Bead ${ }_{\mathbf{A T P}} \mathbf{D}_{\mathbf{7}}$ is derivatized with a structure switching aptamer that senses ATP. In the initial state, the aptamer is complexed with a Cy5-labeled oligonucleotide (7), which is complementary to one of its stems. Bead ${ }_{8} \mathbf{D}_{\mathbf{4}}$ are covered with enzyme and Cy5-labeled substrate $\left(\mathbf{E}_{\mathbf{1}}\right.$ cleaves $\mathbf{S}_{\mathbf{2}}$ on $\left.{ }_{\mathbf{8}} \mathbf{D}_{\mathbf{4}}\right)$. On the ${ }_{\mathbf{8}} \mathbf{D}_{\mathbf{4}}$ bead substrate is blocked with 8c complementary to $\mathbf{8}$ (Figure 4). On the ${ }_{7} \mathbf{A N D}_{\mathbf{4}} \rightarrow \mathbf{D}_{\mathbf{5}}$ bead enzyme is blocked with an inhibitory oligonucleotide complementary to $\mathbf{7}$ (2c in Figure 4) and substrate is blocked with $\mathbf{4 c}$ complementary to 4 (Figure 4). On the surface of ${ }_{\mathbf{A T P}} \mathbf{D}_{7}$ beads upon recognition of ATP, there is stabilization of the ATPbinding site, which the increases rate of the release of $\mathbf{7}$, and decreases the rate of the recapture of 7 to the same beads. Oligonucleotide 7 diffuses and binds to its complement (the acceptor oligonucleotide 2c). The ${ }_{\mathbf{8}} \mathbf{D}_{\mathbf{4}}$ bead is activated through a strand-displacement-based recognition: $\mathbf{8}$ recognizes $\mathbf{8 c}$ on ${ }_{\mathbf{8}} \mathbf{D}_{\mathbf{4}}$. These input trigger the catalytic release of oligonucleotide 4 (Cy5-labeled). Both oligonucleotides 7 and 4 then serve as inputs for a hub bead. The hub performs an AND logic with $\mathbf{7}$ and $\mathbf{4}$ as inputs, and oligonucleotide $\mathbf{5}$ as an output $\left({ }_{7} \mathbf{A} \mathbf{N D}_{\mathbf{4}} \rightarrow \mathbf{D}_{\mathbf{5}}, 5.8 \mu \mathrm{m}\right)$ through the following chemistry: $\mathbf{7}$ activates enzyme $\mathbf{E}_{\mathbf{2}}$ and $\mathbf{4}$ activates substrate $\mathbf{S}_{\mathbf{3}}$ through strand displacement (Figure 4). In the final step of this network, oligonucleotide $\mathbf{5}$ is captured by $\mathbf{5} \mathbf{A}$ bead $(8.2 \mu \mathrm{m})$, which shows approximately twofold increase in fluorescence, only if the AND hub is active. 